$p \mathrm{H} 6,3$ et 6,5 ; en cas de lait neutralisé, on ne constate au contraire aucune coagulation, la masse reste homogène pendant plusieurs jours et, par filtration, on ne peut obtenir qu'un sérum trouble et laiteux dont le $p \mathrm{H}$ s'élève au-dessus de 6,5 et pouvant même atteindre 8,5 en cas d'une addition relativement forte de carbonate ou bicarbonate de sodium. Cette méthode que j'ai eu l'occasion d'expérimenter sur un grand nombre d'échantillons de lait donne d'excellents résultats, mais seulement au point de vue de la détection des laits neutralisés.

La formation du coagulum peut cependant, dans certains cas, provoquer un doute. En effet, dans un lait fraîchement trait, la coagulation est ténue et elle augmente à mesure que l'acidité croît, car elle est liée à la présence d'acides libres (acide carbonique et acide lactique) ; et, pour ne pas se produire dans un lait carbonaté, il faut que l'alealinisation ait été suffisante pour neutraliser cette acidité, la présence de faibles quantités d'acides libres permet encore une coagulation partielle. Mais la détermination de l'acidité libre de sérum permet de réduire ee doute à néant, un $p H$ plus élevé que 6,5 étant toujours un indice de neutralisation ; par exemple, le sérum méthylique d'un lait additionné de 0 gr. 2 par litre de carbonate de sodium accuse un $p \mathrm{H}$ de 6,8 ; si on désire opérer rapidement, on peut déterminer cette acidité libre à l'aide de l'indicateur universel de Merck.

Nous avons donc ainsi, à notre disposition, une méthode excellente de détection et une autre d'analyse qui nous permettent de retrouver avec certitude la moindre addition de carbonate ou de bicarbonate de sodium au lait.

\title{
REVUE
}

\section{ENTRETIEN ET NETTOYAGE DES MACHINES A TRAIRE}

par

\author{
G. GÉNIN
}

Ingénieur chimiste E. P. C.

L'emploi des machines à traire n'a pas encore fait de très grands progrès en France, alors qu'aux Etats-Unis, en Australie, en Nouvelle-Zélande et même en Grande-Bretagne, ces machines sont très répandues dans le monde agricole. C'est ainsi qu'en Nouvelle Zélandec il a été établi que sur 4 fermes, 3 sont munies de machines à traire.

Il faut néanmoins espérer que l'emploi de ces machines ira en se 
développant et qu'elles deviendront un jour aussi répandues que les écrémeuses. Si, pendant longtemps, ces machines se sont révélées imparfaites et si même parfois leur emploi présentait de graves dangers de contamination du lait, on peut estimer aujourd'hui que ces inconvénients ont été surmontés et la machine à traire moderne, de très grande efficacité, est capable, si elle est convenablement entretenue et nettoyée, de fournir un lait parfait et de qualité de conservation excellente.

Il faut néanmoins pour obtenir ces résultats que l'entretien en soit parfaitement assuré et la présence dans les machines à traire d'un certain nombre de pièces en caoutchouc pose certains problèmes dont il importe de souligner l'importance. C'est la raison pour laquelle nous ferons appel dans cette étude aux conseils très judicieux donnés dans une publication de l'Association anglaise des planteurs de eaoutchoue, établie à l'usage des agriculteurs anglais.

\section{Les différentes parties en caoutchouc de la machine à traire.}

Les deux principales difficultés qui résultaient de l'emploi des anciennes machines à traire ont été aujourd'hui complètement surmontées. Ces difficultés étaient dues à ce que jadis, il était pour ainsi dire impossible d'obtenir un caoutchoue résistant d'une faȩon parfaite à l'action du lait. En outre, on n'était pas parvenu à assurer la fabrication d'un caoutchouc capable de résister pendant un temps très prolongé à des stérilisations fréquemment renouvelées. On peut dire aujourd'hui que l'étude scientifique des mélanges de caoutchouc destinés à la fabrication des différentes parties des machines à traire a résolu ces deux problèmes essentiels.

\section{Précautions à prendre au cours de la traite.}

Il est bien évident qu'il faut prendre pendant la traite d'un animal avec une machine à traire, les mêmes précautions que celles qui se sont révélées indispensables lorsqu'on opère à la main. C'est ainsi que les flancs de l'animal et le pis doivent être parfaitement nettoyés et que les trayons doivent être séchés après lavage pour qu'ils ne retiennent pas quelques gouttes d'eau qui risqueraient de passer dans le lait au cours de la traite.

Avant d'appliquer les trayeurs, il faut également éliminer les premières gouttes de lait qui ne doivent pas être mélangées au lait normal et vérifier que toute la machine est en parfait état et qu'en particulier les liaisons entre tubes et les différentes parties de la machine sont bien assurées.

\section{Les maladies du pis de la vache.}

La présence d'organismes pathogènes dans le pis est une question 
qui doit faire l'objet de tous les soins de l'agriculteur et il est intéressant de rappeler que pendant un certain temps, on a admis que les machines à traire favorisaient le développement de la mastite dans les troupeaux de vaches laitières. M. Edwards, de l'Institut de recherches laitières Hannah, a même écrit que l'emploi des machines pouvait contribuer au développement de la mastite. Il semble pourtant, d'après de nombreuses statistiques établies dans les régions où les machines à traire sont très employées, que le développement de la mastite n'est pas du tout plus important que dans les régions où la traite à la main est en vigueur. Il est probable que c'est un emploi des machines effectué dans des conditions défectueuses qui a conduit à faire admettre que les machines à traire favorisaient le développement de la mastite.

En fait, dès qu'un animal présente les symptômes d'infection du pis, il est indispensable de le traire à la main, afin d'éviter toute contagion. Si cette précaution est prise et si l'on prend soin de nettoyer et de stériliser la machine, comme nous le verrons plus loin, tout risque de contagion est évité.

\section{Nettoyage et stérilisation des machines à traire.}

Il est aujourd'hui établi que le bon rendement d'une machine à traire dépend uniquement du soin apporté à son entretien et à son nettoyage. De nombreuses études ont montré que si les indications que nous allons exposer sont suivies, on peut obtenir sans aucun risque du lait de qualité parfaite :

La machine doit être soigneusement nettoyée après chaque traite et ce nettoyage doit avoir lieu sitôt que la traite est terminée. Il est en effet extrêmement difficile d'éliminer les particules de lait qui ont pu sécher sur la surface du caoutchoue et cela active son usure et sa détérioration, à moins que par négligence on laisse subsister ces résidus, ce qui entraîne la contamination du lait.

Voici les conseils que donnent MM. Mackintosh et Matтick, de l'Institut national des recherches laitières de Reading, conseils qui ont été publiés en détail dans le Bulletin du Ministère de l'Agriculture. Dès que la traite est terminée, on aspire dans toute la machine de l'eau froide, en plongeant les trayeurs dans un seau rempli d'eau et en faisant fonctionner la pompe. Il faut toutefois toutes les 5 secondes sortir les trayeurs du seau afin qu'une certaine quantité d'air pénètre dans la machine, le brassage qui résulte de la présence d'air et d'eau assurant une meilleure élimination des sédiments.

On répète alors ce nettoyage mais cette fois avec de l'eau chaude à $80^{\circ}$ et on brosse l'intérieur des trayeurs et des tubes de caoutchouc. Toutes ces opérations doivent être faites avec beaucoup de soin et cette fois, il ne faut pas aspirer de l'air, ee qui aurait pour effet de 
refroidir l'eau. On rince à nouveau toutes les parties de la machine dans de l'eau chaude, et on les laisse égoutter avant de les stériliser. La stérilisation s'effectue au moyen de vapeur et elle doit durer 15 minutes. On peut soit introduire la machine complètement montée dans le récipient où se fait la stérilisation, soit au contraire introduire les différentes parties de l'appareil après démontage. On suspendra par exemple les différentes pièces de la machine à traire dans un sac mousseline, qui sera placé à l'intérieur du stérilisateur, de façon à éviter tout contact entre le métal et le caoutchouc et réduire l'usure et la dégradation du caoutchouc. Toutes les pièces de la machine, qu'elles soient en caoutchouc ou en métal, doivent être stérilisées, à l'exception du pulsateur.

\section{Une autre méthode de stérilisation.}

Depuis quelques années, une autre méthode de nettoyage des machines à traire par la soude caustique et l'eau bouillante est employée en Nouvelle-Zélande et semble donner entière satisfaction. Il a même été écrit dans une revue d'agriculture qu'aucune autre méthode connue ne peut se comparer avec celle-ci, au point de vue de la simplicité, de l'efficacité, de la rapidité et du bon marché.

Avec les pièces en caoutchouc d'excellente qualité dont la surface est parfaitement lisse, il y a danger de détérioration, lorsqu'on utilise pour les brosser des dispositifs métalliques en formes de spirale ou de grattoir. Au contraire, l'emploi de soude caustique, en combinaison avec l'eau bouillante, n'entraîne aucune dégradation du caoutchouc et permet immédiatement d'éliminer toute trace de matière grasse. Le caoutchouc soumis à ce traitement acquiert une surface parfaitement lisse et sa durée est considérablement augmentée. Il est toutefois important de régler convenablement la concentration de la solution caustique et par exemple de verser dans une dizaine de litres d'eau une cuillère à café de soude caustique. Après ce traitement, on rince à l'eau bouillante, puis à l'eau froide.

\section{L'emploi des désinfectants chimiques.}

On a souvent conseillé l'emploi de produits chimiques et de produits désinfectants pour nettoyer et désinfecter les machines à traire. On ne peut être certain que ces produits aient donné de bons résultats. En effet, lorsqu'on utilise des désinfectants ou des produits chimiques, il est presque impossible d'éliminer complètement toute trace de ces produits de la machine à traire et on risque done d'en trouver dans le lait avec tous les inconvénients qui en résultent.

En outre, les parties métalliques de la machine à traire sont susceptibles d'être corrodées par ces produits chimiques. Le Collège d'agriculture de Californie a également montré que les désinfectants 
à base de chlore sont susceptibles de réduire considérablement la durée des partíes en caoutchouc; aussi est-il préférable de s'abstenir d'employer des produits de ce genre.

Nous espérons que ces quelques indications aideront à modifier certaines opinions inexactes concernant l'utilité des machines à traire et faciliteront l'emploi de ces machines dans les installations agricoles françaises, d'autant plus que l'électrification des campagnes se développant chaque année, il devient de plus en plus facile de trouver dans la ferme l'énergie électrique nécessaire au fonctionnement des différents appareils automatiques.

\section{BIBLIOGRAPHIE ANALYTIQUE}

\section{LES LIVRES}

\section{Sirks (H. A.). - Etude des propriétés qui peuvent servir à} distinguer les poudres de lait des poudres de beurre. Vereeniging tot Exploitatie eener Proefzuivelboerderij te Hoorn. Verslag over het jaar 1933 (Association pour l'exploitation d'une ferme d'essais de laiterie à Hoorn. Rapport de l'année 1933), p. $1-16$.

Le chauffage et le séchage produisant des modifications considérables dans la dimension des globules de matière grasse, il a paru impossible de distinguer les poudres de lait des poudres de beurre par une méthode basée sur la distribution probablement différente, en raison de leurs dimensions, des globules de matière grasse dans les liquides originaux, e'est-à-dire le lait et une émulsion de matière grasse de beurre dans du lait écrémé.

Si les poudres de beurre ont été préparées avec du vieux beurre de qualité inférieure, on les décelera en utilisant les épreuves de Kreis et de Fellenberg (suiffage et rancidité); mais si ce n'est pas le cas, les matières grasses des poudres fraîchement préparées ne réagissent généralement pas. Elles ne réagissent, du reste, que très faiblement, même si on les eonserve pendant trés longtemps.

HañS a observé que le chauffage avec un alcali et de la glycérine change une matière grasse de beurre altérée en une masse brune à consistance de gelée. On peut utiliser cette observation dans quelques cas pour déceler de la poudre de beurre. L'examen d'un certain nombre de poudres de beurre, dont la matière grasse ne donnait pas lieu à la réaction de Hanus, a montré que, dans la plupart des cas, la saponification de la matièrè grasse était accompagnée d'une formation continuelle de mousse, très apparente et anormalement forte.

En chauffant plusieurs poudres de lait et poudres de beurre à $103-105^{\circ} \mathrm{C}$. pendant 2 ou 3 heures, on constata que l'assombrissement de la teinte jaunâtre qui accompagne ce chauffage était généralement plus apparent dans les poudres de beurre que dans les poudres de lait. L'observation, dans des poudres d'origine inconnue, des caractéristiques indiquées dans le présent 